\title{
Top quark pair-production cross section measurements at the LHC
}

\section{Sergio Grancagnolo*, on behalf of the ATLAS and CMS Collaborations.}

Humboldt University Berlin

E-mail: sergio.grancagnolo@cern.ch

Latest results on inclusive top quark pair-production cross sections are presented using protonproton collision data collected at the LHC. The inclusive measurements reach high precision and are compared to the best available theoretical calculations. Differential measurements of the kinematic properties of the top quark production are also discussed. These measurements from ATLAS and CMS experiments probe our understanding of top quark pair production in the $13 \mathrm{TeV}$ regime. The results, unfolded to particle and parton level, are compared to predictions of Monte Carlo generators implementing NLO matrix elements matched with parton showers and NNLO QCD theory calculations.

XXVII International Workshop on Deep-Inelastic Scattering and Related Subjects - DIS2019

8-12 April, 2019

Torino, Italy

${ }^{*}$ Speaker. 


\section{Introduction}

The top $(t)$ quark is the heaviest particle in the Standard Model (SM), and it is produced in abundance in proton-proton $(p p)$ collisions at the LHC [1]. The top quark couples to all gauge bosons and the Higgs boson, allowing experiments to test the SM, constrain input parameters to quantum chromodynamics (QCD) predictions, and probe beyond-SM scenarios. About $8 t \bar{t}$ pairs are produced every second at a luminosity of $10^{34} \mathrm{~cm}^{-2} \mathrm{~s}^{-1}$ at $13 \mathrm{TeV}$. Each of the two top quarks decays into a bottom $(b)$ quark and a $W$ boson, with a branching ratio close to $100 \%$, in a final state that can be classified according to the two $W$ boson decays: a $t \bar{t}$ event is expected to end $9 \%$ of times in two charged leptons, and $45 \%$ in a single charged lepton plus jets. The production cross section $\left(\sigma_{t \bar{t}}\right)$ can be measured with different methods, either in the acceptance of the detector (fiducial) or extrapolated to the full phase space. It can be measured as a function of one observable (differential) or more observables (multidifferential), as an absolute value or normalised to the inclusive $\sigma_{t \bar{t}}$ in the same phase space. Experimental observables are compared to the output of various Monte Carlo (MC) generators and tunes, implementing matrix element (ME) calculations at the next-toleading order (NLO), simulating higher orders through parton showering (PS). Comparison with predictions, generated after full ME and PS simulation, including QCD and electroweak radiation, can be performed at the level of partons (e.g. quarks before hadronisation) or quasi-stable particles.

The CMS experiment [2] used channels with two leptons $(e e, e \mu, \mu \mu)$ to perform measurements of the $t \bar{t}$ differential, multi-differential, and total cross sections using $35.9 \mathrm{fb}^{-1}$ of data at $13 \mathrm{TeV}$. The ATLAS experiment [3] measured fiducial and differential cross sections of $t \bar{t}$ production in addition to $b$-flavored jets ( $b$-jets), in the $e \mu$ dilepton channel and in channels with one lepton $(e, \mu)$ plus jets in $36.1 \mathrm{fb}^{-1}$ of data at $13 \mathrm{TeV}$.

\section{CMS Results}

For one measurement of the $t \bar{t}$ production cross section [4], CMS selected events with at least two oppositely-charged isolated leptons $(e, \mu)$ of high transverse momentum $\left(p_{\mathrm{T}}\right)$, including those coming from a $\tau$ lepton decay. Events are classified according to lepton flavor, and to the number of jets, counting separately those coming from light quarks and those coming from $b$-quarks. Jets are $b$-tagged with high purity ( $0.1 \%$ of light jets mistagged) with an efficiency of $41 \%$. Such a categorization makes it possible to constrain systematic uncertainties due to theoretical modelling (e.g. those coming from variations in scales for ME and PS matching), while performing a template fit, to estimate signal and background contributions in each channel. The uncertainty on the visible fiducial cross section $\left(\sigma_{t \bar{t}}^{\mathrm{vis}}\right)$ is $3.8 \%$ with a contribution from lepton isolation and identification of $2 \%$ and from parton distribution functions (PDF) of $1.1 \%$. The fiducial cross section is then extrapolated to the full phase space for a final result of $\sigma_{t \bar{t}}=803 \pm 2$ (stat) \pm 25 (syst) \pm 20 (lumi) pb. A summary of LHC and Tevatron measurements as a function of the centre-of-mass energy, compared to the next-to-NLO (NNLO) QCD calculation complemented with next-to-next-to-leading$\log$ (NNLL) resummation, is shown in Fig. 1 [5].

Events containing exactly two oppositely charged leptons ( $e, \mu$ not from $\tau$ or $b$ ); at least two jets, at least one $b$-tagged with high efficiency $(79-87 \% b$-tagging, $10 \%$ light-flavor contamination); and missing transverse momentum larger than $40 \mathrm{GeV}$ are used to measure $t \bar{t}$ differential 


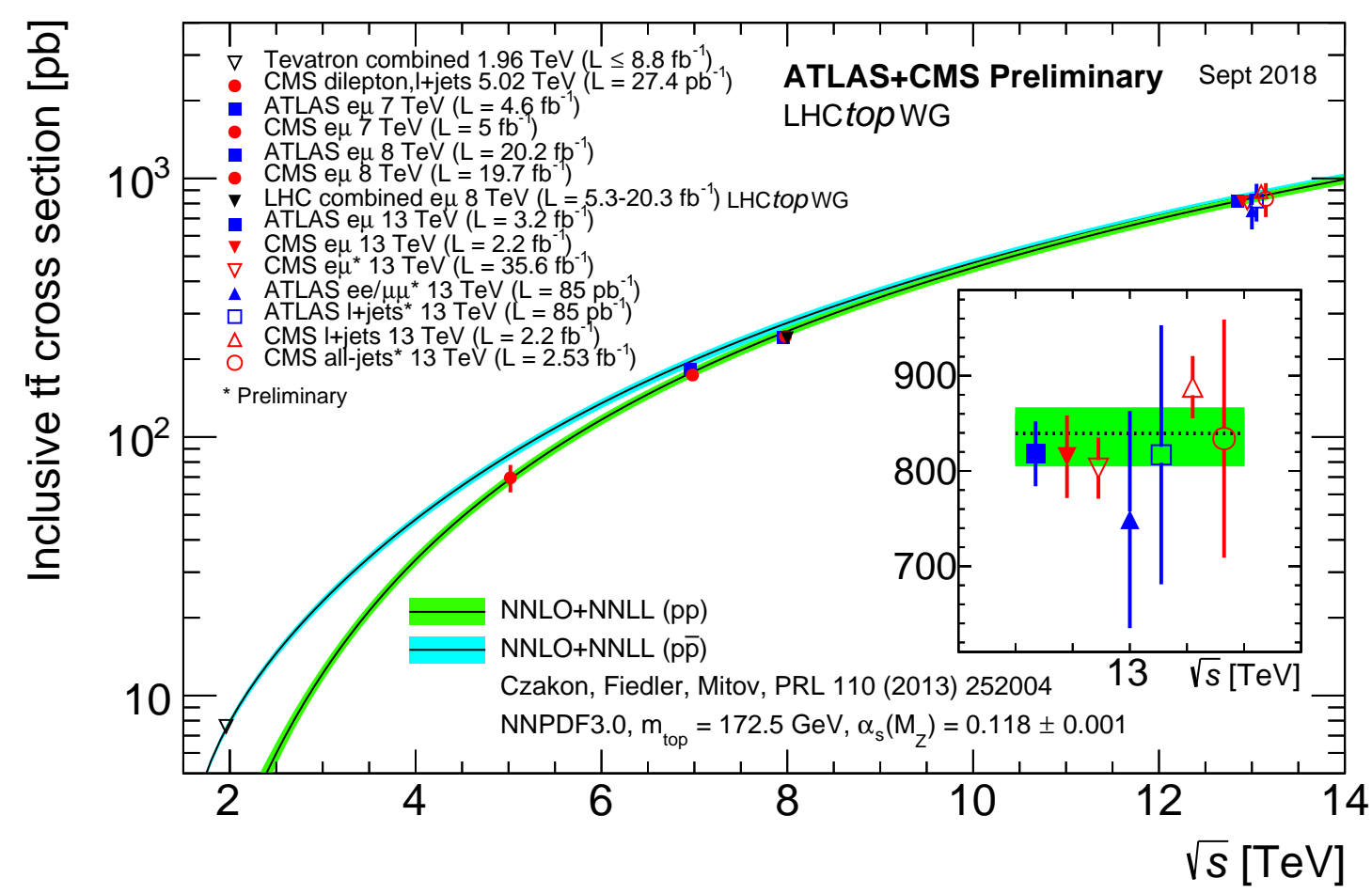

Figure 1: Summary of LHC and Tevatron measurements of the top-pair production cross section as a function of the centre-of-mass energy, compared to predictions (quoted at $m_{t}=172.5 \mathrm{GeV}$ ) [5].

cross sections as a function of several kinematic variables [6]. A kinematic reconstruction is performed to assign reconstructed objects to the corresponding originally-generated decay products, with an efficiency of $90 \%$. For a given observable $X$, the absolute cross-section distribution is obtained via regularised unfolding: signal events are background subtracted and corrected for detector efficiencies and acceptance, as well as for bin migration. The bin width is optimised for purity and stability (fraction of reconstructed events matching corresponding generated events in a given bin, and vice versa). The purity and stability are typically at the level of $50 \%$. Normalising to the sum of all $X$ bins avoid dependence on the total inclusive $\sigma_{t \bar{t}}$ used in MC. Examples of results are shown in Fig. 2. The normalised $\sigma_{t \bar{t}}$ at parton level, using the rapidity difference between $t$ and $\bar{t}$ quarks, is shown on the left side. This distribution allows the extraction of charge asymmetries, which are well modelled by theoretical predictions, as are morst of the measured differential cross sections. The absolute $\sigma_{t \bar{t}}$ at particle level, using the leading lepton $p_{\mathrm{T}}^{\ell}$, is shown on the right side. This distribution can be used to test $t$-quark decay model-ling and spin correlations among the two finalstate leptons. Significant disagreement is noted for this and other observables, between data and simulation at NLO precision in QCD. Predictions beyond-NLO precisions are generally in closer agreement, although some discrepancies remain.

With the same event selection and unfolding strategy (with purity and stability above 20\%), measurements of $t \bar{t}$ cross section are performed also as a function of pair of variables (doubledifferential) and in bins of number of jets $N_{\text {jet }}$, invariant mass $M(t \bar{t})$ and rapidity $y(t \bar{t})$ of the $t \bar{t}$ 

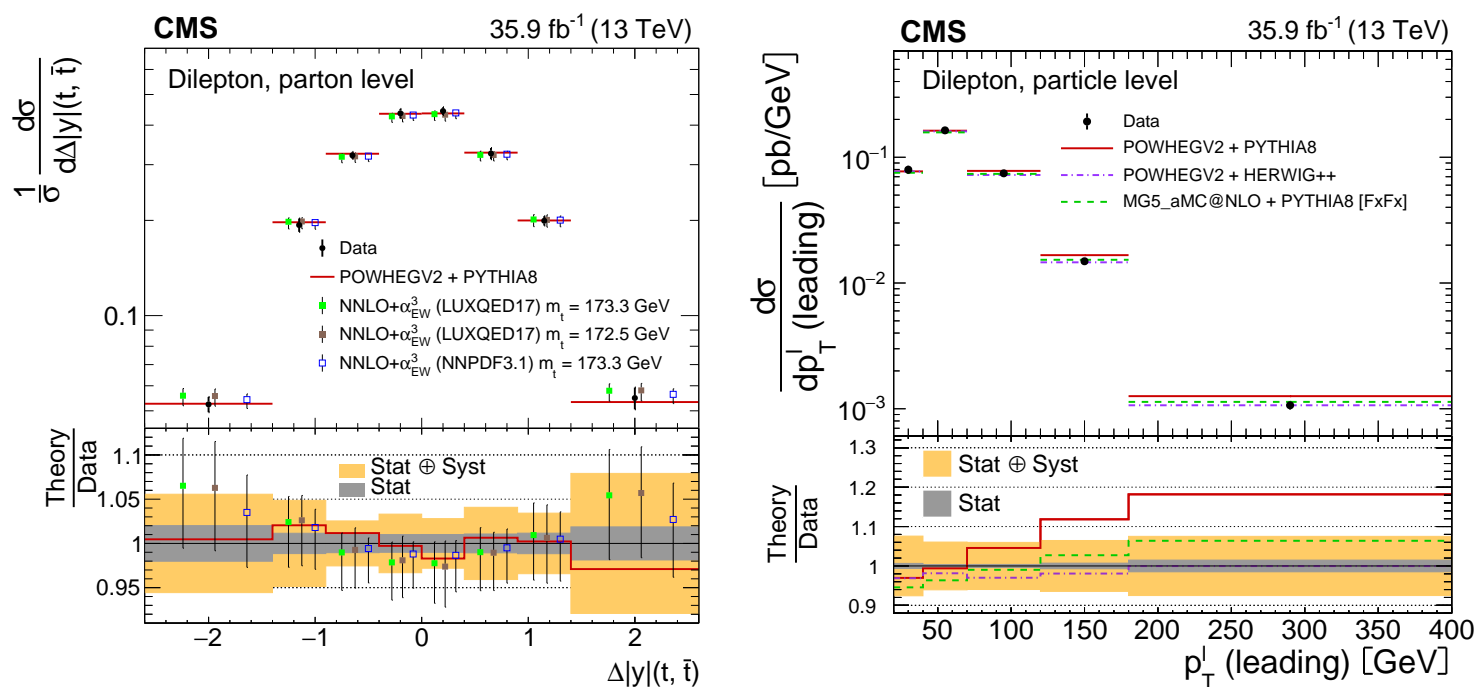

Figure 2: Left: Parton-level normalised cross section as a function of $\Delta|y|(t, \bar{t})$ compared with NNLO predictions. Right: Particle-level absolute cross section as a function of leading lepton $p_{\mathrm{T}}^{\ell}$ compared with NLO predictions [6].

system (triple-differential) [7]. An example is shown in Fig. 3, for the variables $p_{\mathrm{T}}(t)$ and $M(t \bar{t})$. Generators generally predict a too-hard $p_{\mathrm{T}}(t)$ distribution, with a larger discrepancy at high $M(t \bar{t})$. Other spectra reveal that none of the tested MC generators is able to provide predictions that correctly describe all distributions across the full set of observables considered.

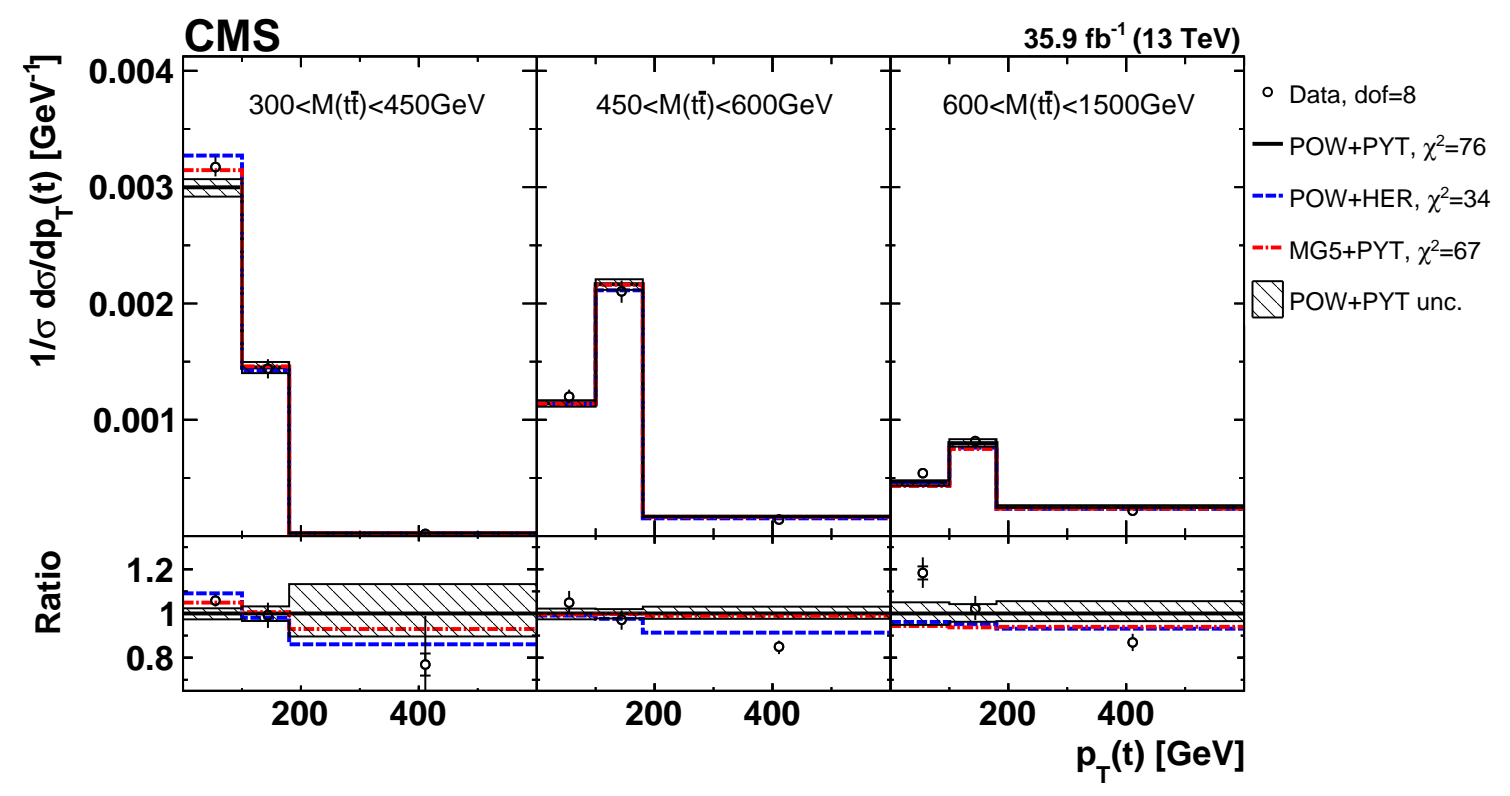

Figure 3: Double differential of $p_{\mathrm{T}}(t)$ in $M(t \bar{t})$ bins [7].

\section{ATLAS Results}

In the ATLAS work presented here [8], the cross section of $t \bar{t}$ production is studied in associ- 

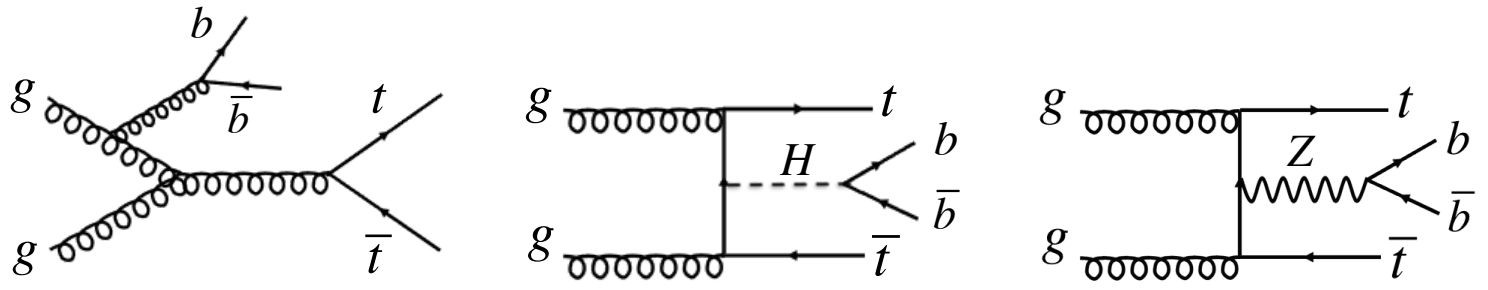

Figure 4: Example Feynman diagrams of processes leading to a $t \bar{t} b \bar{b}$ final state [8].

ation with $b$-jets. Such a measurement is important for several reasons. Calculating the amplitude of the process in the left side of Fig. 4 is complicated by the uncertainties due to the non-negligible $b$-quark mass. This is also the main background to the $t \bar{t} H$ process, which allows direct determination of the $t$-quark Yukawa coupling, when considering the dominating decay channel $H \rightarrow b \bar{b}$ (Fig. 4, middle). Also other processes, like $t \bar{t}$ in association with a vector boson (Fig. 4, right) are probed in related measurements.

Events are selected in two decay channels: dilepton and lepton plus jets. In the first, they contain exactly two isolated high- $p_{\mathrm{T}}$ leptons (one $e$ and one $\mu$ ) of opposite charge and at least two jets, at least two of which are $b$-tagged (77\% efficiency working point). In the second channel, events contain exactly one charged lepton as defined above and at least five jets, at least two of which are $b$-tagged (60\% efficiency working point). In both cases, after baseline selection, the prediction underestimates the observed data for events with more than two $b$-tagged jets. A correction is then applied to account for $c$-jets and light jets predictions, with data-driven scale factors. The measured inclusive fiducial cross sections are shown in Fig. 5. They generally exceed the $t \bar{t} b \bar{b}$ predictions
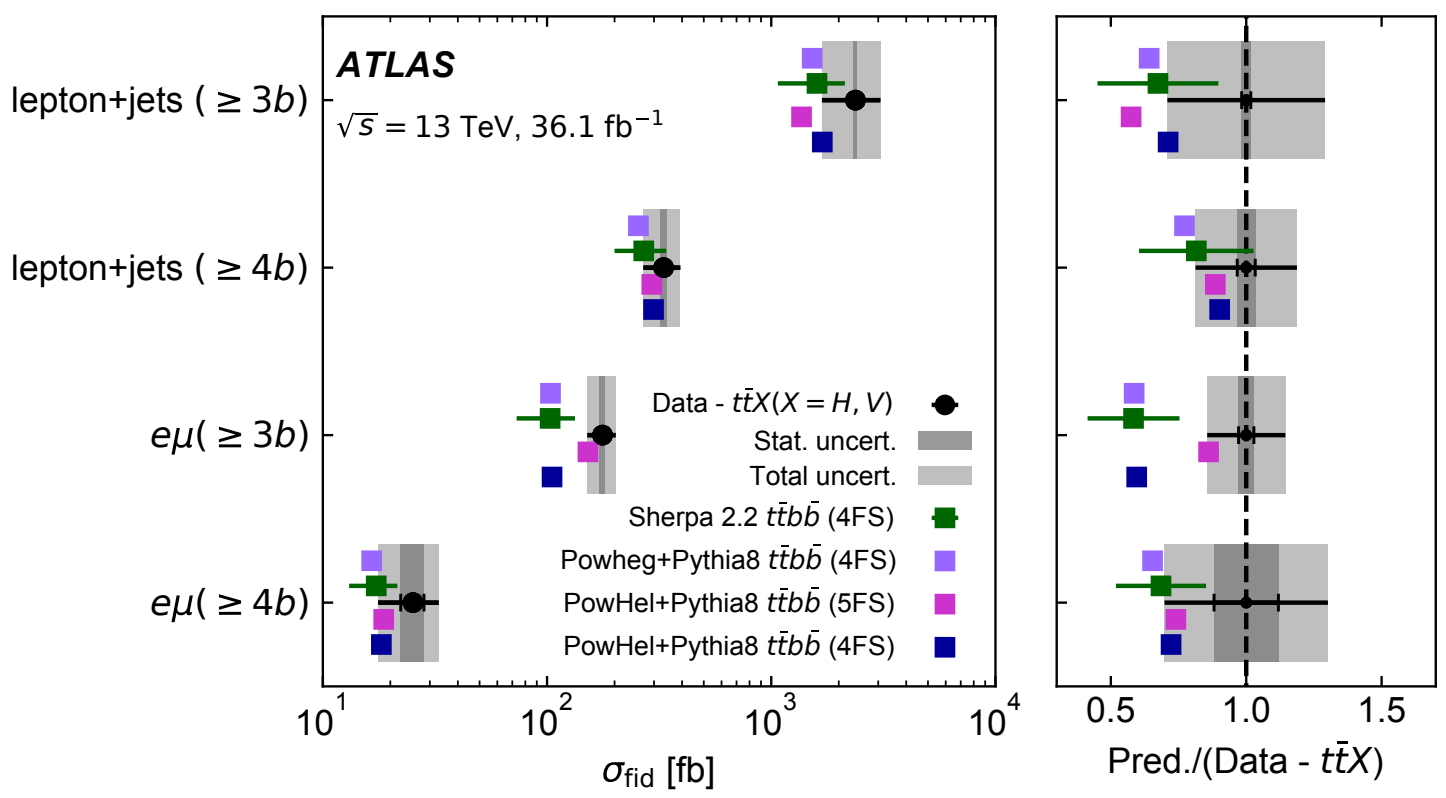

Figure 5: The measured $t \bar{t} b \bar{b}$ fiducial cross sections, compared with predictions [8].

from various NLO ME calculations matched to a PS, but are compatible within the total uncertain- 
ties. Experimental uncertainties are smaller than those in the predictions. To obtain differential cross sections as a function of global event properties and properties of $b$-jet pairs, measured distributions are unfolded at particle level, correcting for resolution effects and detector efficiencies and acceptance. Good agreement with unfolded data is found for most theoretical predictions when compared with the differential measurements.

\section{Summary}

Using $36 \mathrm{fb}^{-1}$ of $p p$ collision data at $13 \mathrm{TeV}$, the ATLAS and CMS experiments have measured inclusive top quark pair-production cross sections. High precision results are compared to the best available theoretical calculations. The total $t \bar{t}$ cross section, extrapolated to the full phase space, is $\sigma_{t \bar{t}}=803 \pm 2$ (stat) \pm 25 (syst) \pm 20 (lumi) pb. Significant disagreements are observed in single and multi-differential $t \bar{t}$ cross sections between data and simulation at NLO precision in QCD. Predictions beyond-NLO precisions are generally in closer agreement, although none of the considered MC generators is able to provide predictions that correctly describe all distributions, across the full spectrum of observables. While being compatible within total uncertainties, the fiducial $t \bar{t} b \bar{b}$ cross section exceeds the predictions from various MC simulations. Good agreement with data is found for most theoretical predictions when compared with the differential measurements of $t \bar{t}$ plus additional $b$-jets.

\section{References}

[1] L. Evans and P. Bryant, LHC Machine, JINST 3 (2008) S08001.

[2] CMS Collaboration, The CMS Experiment at the CERN LHC, JINST 3 (2008) S08004.

[3] ATLAS Collaboration, The ATLAS Experiment at the CERN Large Hadron Collider, JINST 3 (2008) S08003.

[4] CMS Collaboration, Measurement of the tit production cross section, the top quark mass, and the strong coupling constant using dilepton events in pp collisions at $\sqrt{s}=13$ TeV, Eur. Phys. J. C79 (2019), 368.

[5] LHC Top WG, Top-pair production cross-section as a function of centre-of-mass energy, [https://twiki.cern.ch/twiki/bin/view/LHCPhysics/LHCTopWG].

[6] CMS Collaboration, Measurements of t⿱亠䒑 differential cross sections in proton-proton collisions at $\sqrt{s}=$ $13 \mathrm{TeV}$ using events containing two leptons, JHEP 02 (2019) 149.

[7] CMS Collaboration, Measurement of tt normalised multi-differential cross sections in pp collisions at $\sqrt{s}=13 \mathrm{TeV}$, and simultaneous determination of the strong coupling strength, top quark pole mass, and parton distribution functions, Submitted to: Eur. Phys. J. (2019).

[8] ATLAS Collaboration, Measurements of inclusive and differential fiducial cross-sections of $t \bar{t}$ production with additional heavy-flavour jets in proton-proton collisions at $\sqrt{s}=13 \mathrm{TeV}$ with the ATLAS detector, JHEP 04 (2019) 046. 